\title{
Sports betting incentives encourage gamblers to select the long odds: An experimental investigation using monetary rewards
}

\author{
MATTHEW J. ROCKLOFF ${ }^{1}$, MATTHEW BROWNE ${ }^{1}$, ALEX M. T. RUSSELL ${ }^{2}$, NERILEE HING ${ }^{1}$ and NANCY GREER ${ }^{3}$
}

${ }^{1}$ Experimental Gambling Research Laboratory, School of Health, Medical and Applied Sciences, CQUniversity, Bundaberg, QLD, Australia ${ }^{2}$ Experimental Gambling Research Laboratory, School of Health, Medical and Applied Sciences, CQUniversity, Sydney, NSW, Australia ${ }^{3}$ Experimental Gambling Research Laboratory, School of Health, Medical and Applied Sciences, CQUniversity, Melbourne, VIC, Australia

(Received: January 18, 2019; revised manuscript received: March 22, 2019; accepted: May 5, 2019)

\begin{abstract}
Background and aims: Incentives for wagering products can provide extra value to gamblers. However, there is no financial reason why this added value should lead people to take greater gambling risks. This study aimed to experimentally test if wagering incentives cause gamblers to choose higher-risk (long odds) bets than un-incentivized bets. Methods: An online experiment was conducted with wagering customers $(N=299$, female $=12)$. Participants bet $\$ 4$ on each of six video game simulations of a sport that they had wagered on in the past 12 months (Australian Football League, Cricket, or Soccer). Each game offered different common wagering incentives: Bonus bet, Better odds/winnings, Reduced risk, Cash rebate, Player's choice of inducement, or No-inducement. For each game, participants could bet on long, medium, or short odds, and subsequently viewed a highlight reel of the simulated game outcome and bet outcome. Results: Participants selected significantly longer odds (i.e., riskier) bets on games when an incentive was offered compared to the No-inducement condition. Better odds/winnings was the most attractive incentive, followed by Bonus bet, Cash rebate, Reduced risk, and No-incentive, respectively. No significant differences were observed based on demographics or problem gambling severity. Discussion and conclusions: The choice of long odds with incentivized bets increases the volatility of player returns. Increased volatility results in more gamblers in a losing position and fewer gamblers with larger wins. Moreover, if long odds bets are priced to provide poorer value to bettors compared to short odds, they would increase gamblers' losses and equivalently increase operators' profits.
\end{abstract}

Keywords: sports betting, inducements, incentives, gambling, wagering

\section{INTRODUCTION}

Inducements offered for sports and race betting have raised concerns due to the potential for deceptive practices, and their potential to alter people's gambling choices in a manner that undermines their best interests (Hing, Sproston, Brook, \& Brading, 2017). Longitudinal studies have shown that exposure to wagering inducements is associated with increased betting expenditure (Browne, Hing, Russell, Thomas, \& Jenkinson, 2019; Hing, Russell, Thomas, \& Jenkinson, 2019; Russell, Hing, Browne, \& Rawat, 2018) and that bettors typically underestimate the cost of using bonus bets (Hing, Browne, et al., 2018). Newall (2015, 2017) also demonstrated that wagering operators tend to most promote and incentivize combined contingencies, which bettors find difficult to calculate, and which are poorly priced relative to their long odds. For any inducement or marketing effort, it is trivial to show that offering greater value should increase consumption. The demand equation is a fundamental tenet of microeconomics, where lowering price - or equivalently increasing value - of a product should translate to more purchases (Friedman, 1949). However, it is also important to consider other aspects of consumption when considering the effects of gambling marketing. Gambling inducements can potentially alter people's risk-based gambling choices and therefore have an additional impact on their spending by encouraging irrational purchases.

One feature of gambling expenditure that impacts on long-run expenditure is the choice of relative risk for bets, that is, short odds versus long odds. Choosing long odds increases the volatility of a gambler's bankroll, where players are more likely across time to be in a sharp winning or losing position. Small losses are more frequent, while wins are less likely - albeit relatively large when they do occur. Among a population of gamblers, an aggregate increase in volatility of play produces more losers, and relatively fewer - and richer - winners. Consequently, even when the pricing of long odds is objectively good, having gamblers persistently choose long odds in aggregate can

\footnotetext{
* Corresponding author: Matthew J. Rockloff, BA, MS, PhD; Experimental Gambling Research Laboratory, School of Health, Medical and Applied Sciences, CQUniversity, Building 408, University Drive (off Isis Highway), Bundaberg, QLD 4670, Australia; Phone: +61 74150 7138; E-mail: m.rockloff@cqu.edu.au
}

This is an open-access article distributed under the terms of the Creative Commons Attribution-NonCommercial 4.0 International License, which permits unrestricted use, distribution, and reproduction in any medium for non-commercial purposes, provided the original author and source are credited, a link to the CC License is provided, and changes - if any - are indicated. 
increase the pool of persons in a losing position. Moreover, when long odds are priced poorly relative to short odds, there is even greater potential that many consumers will be worse off, as the greater profits flow to bookmakers (Newall, 2015).

This study aimed to test for the effects of inducements on gamblers' overall propensity to accept greater risks on their bets. In addition, we speculated that those with gambling problems [problem gambling severity index (PGSI); Ferris \& Wynne, 2001] would be more likely to make riskier bets as an observable consequence of being offered an inducement. This speculation is based on previous research finding that higher-risk gamblers report more influence from gambling advertising (Binde, 2014), including for wagering (Hing, Russell, Lamont, \& Vitartas, 2017). The study also sought to identify whether, and how, different common wagering inducements, including Bonus bets, Better odds/winnings, Reduced risk, and Cash rebate (described in more detail in Methods below), might change people's choice of long versus short odds bets. The following research questions were addressed:

Q1: Do inducements influence the selection of longer odds bets?

Q2: When given a choice, which inducements do sports bettors select?

Q3: What inducements do participants rank as most attractive?

This research was devised to draw conclusions about the potential causal role that wagering inducements play in sports betting. By testing for inducements with the highest likelihood of causing or exacerbating harm, the study can inform policy and regulations aimed at reforming their provision, marketing, and structural characteristics. While previous studies have analyzed bettors' exposure to wagering inducements, their structural characteristics, and their association with more, and more impulsive, betting (Hing, Russell, Li, \& Vitartas, 2018; Hing et al., 2019; Hing, Sproston, et al., 2017; Lopez-Gonzalez, Estévez, \& Griffiths, 2017, 2018a, 2018b; Lopez-Gonzalez \& Griffiths, 2017), this is the first study to test their effects on risk-taking using an experimental design.

\section{METHODS}

\section{Recruitment and sampling}

Inclusion criteria were residing in the state of Victoria, Australia; aged 18 years or older; and betting on Australian Football League (AFL), cricket, or soccer at least twice within the past 12 months.

Two major sports betting companies operating in Australia assisted recruitment by e-mailing their customers an invitation to complete the survey, preferentially e-mailing their most frequent sports bettors first. The invitation contained a weblink to the information sheet, consent form, and online survey. The survey took about 15-20 min to complete, and most finished in a one-time sitting. Only 12 participants took longer than $100 \mathrm{~min}$ to finish, and all but two completed the survey in the same day. The betting companies knew about, but had no control over, the nature and purpose of the research, nor any access to the resultant data. We designed the sampling procedure and have no reason to think the betting companies manipulated this direction, especially given the high proportions of respondents who met criteria for problem and at-risk gambling.

The soft launch (May 9-16, 2017), which consisted only of Operator 1's customers, yielded 34 responses of which 28 had complete data that were included in analyses. The hard launch (June 1-18, 2017) yielded 386 responses, of which 271 were used in analyses. The remaining 115 were discarded because 4 did not live in Victoria, 1 had not bet on any sports in the past 12 months, and 110 provided incomplete responses. The final data set contained 299 responses. We aimed to recruit a large pool of regular sports bettors. Recruiting first the most frequent sports bettors provided $186(62 \%)$ at least fortnightly bettors, whereas the remaining $113(38 \%)$ were less than fortnightly bettors.

Only one modification was made to the survey after the soft launch. For the participant's sixth game, which involved them selecting an inducement condition, we removed the Better odds/winnings option during the hard launch. This modification is described and justified later.

\section{Participants}

Most of the 299 respondents were English-speaking males (male $=287)$, aged $18-39$ years $(M=36.0, S D=13.7)$. Non-problem gambler (NPG) accounted for $15.7 \%(n=47)$, low risk (LR) $27.4 \%(n=82)$, moderate risk (MR) $38.1 \%$ $(n=114)$, and problem gambler $(\mathrm{PG}) 18.7 \%(n=56)$.

\section{Measures}

The combined online survey and experiment took approximately $20 \mathrm{~min}$ and had several components.

Screening. Participants were asked what sports, including AFL, soccer, and cricket, they had bet on in the past 12 months. Participants were serially assigned to bet on the lowest quota-filled sport, as long as they had also bet on that sport within the past 12 months.

\section{Demographics}

Age, gender, postcode, main language spoken at home, annual household income, and annual personal income were asked.

Problem gambling severity. This was assessed using the PGSI (Ferris \& Wynne, 2001), which contains nine items with four response options ("never" $=0$, "sometimes" $=1$, "most of the time" $=2$, and "almost always" =3). Total summed scores range between 0 and 27 , where $0=$ nonproblem gambling, $1-2=$ low risk, 3-7 = moderate risk, and $8-27=$ problem gambling.

Betting-risk choices. Participants were required to bet from a choice of short odds, medium odds, or long odds on six games, representing different "inducement" types (see below). The final question required participants to rank different inducement types from "most attractive" to "least attractive." 


\section{Procedure}

The experimental paradigm used video game technology to simulate highlight reels for AFL, cricket, and soccer. Modern video game technology produces highly engaging simulations of these sports. Outputs from commercially available 3D video games were used to generate highlight reels of simulated matches in a consistent format, which were presented to participants online to display the results/ outcomes of each bet (Figure 1). Audio of an announcer was added to videos with standard commentary, including which team won the toss and who was ahead at half-time, which was relevant for some of the inducements.

Wagering on six simulated games with varying incentives. After completing the demographic and sports betting questions, participants were asked to bet on six trials, including one for each of the four inducements and one for the No-inducement condition (Games 1-5), as well as one trial to assess the relative attractiveness of the mostfavored inducements (Game 6). For Games 1-6, participants selected one of the two competing teams to back and chose 1 of 3 risk levels for their bet (short, medium, or long odds), with both teams priced identically. For Game 6, participants selected one of the inducement conditions for their bet, including the No-inducement condition. For all six games, participants bet with real money (\$4) provided by the research team and took home any winnings at the end of the experiment.

Games 1-5. Each participant viewed betting odds (always evenly matched), placed bets, watched a highlight reel, and was paid any winnings for the first five games, each consisting of four inducement trials and one no-inducement trial. We simulated real teams that were approximately evenly matched for a win, based on competition ladders, during the time of the experiment to enhance the validity of offering balanced odds for each match-up.

The order of the offered inducements and team matchups was randomized to avoid order effects. No team was involved in more than one match. Players were provided with a stake (\$4) for each trial, with winnings not carried over into subsequent trials to minimize carryover effects. Instead, winnings directly accrued to the player's "account" and contributed to their take-home compensation. Each trial involved the player being offered different betting options, which varied in the degree of risk and that had an inversely proportional potential payoff (i.e., short, medium, or long odds). The tendency of players to gamble relatively long odds represented (behavioral and repeated measures) the key-dependent variable in the experiment.

For this study, four common classes of incentives were extracted from the 15 generic types of wagering inducements identified by Hing, Sproston, Brading, and Brook (2015). The incentives studied in this experiment comprised: Bonus bets, Better odds/winnings, Reduced risk, and Cash rebate. These "incentives" distil the important attractive features behind the varying inducements offered by operators in Australia.

Hing et al. (2015) noted that the broad class of the incentive, rather than the specific mechanics of the inducement, is likely most important in influencing player choices. Therefore, the experiment incorporated specific inducements to represent the four different classes of incentives, rather than attempting an impractical task of modeling of all 15 inducements. Table 1 identifies the chosen simulation of these inducements.

Participants chose one of the two competing teams to win the match and simultaneously selected the level of risk (short, medium, or long odds) they would take on the bet. Bets were each made for a fixed amount of cash (\$4). Immediately after each bet, the participant viewed the highlight reel of each game to learn the outcome. Total compensation varied based on bet type and betting success, and paid as an electronic shopping voucher. Total payments ranged from $\$ 24$ to $\$ 84.80$ inclusive of Game 6 . To maintain goodwill, we set minimum compensation at $\$ 24$, although we did not notify participants of this fact until after they had completed the experiment.

The risk or odds for participants' bets was determined by the schedule outlined in Table 2. Since all teams were evenly matched for a win, an outright win was chosen as the short-odds bet, whereas other longer odds bets required the player's chosen team to win by a larger margin (more points, goals, or wickets). All conditions had the same base $\$$ win outcomes for short, medium, and long odds bets, which increased in $\$$ won by risk level (Table 2). The $\$$ amounts that could be won for a long odds bet were similar to what might be expected from a commercial operator
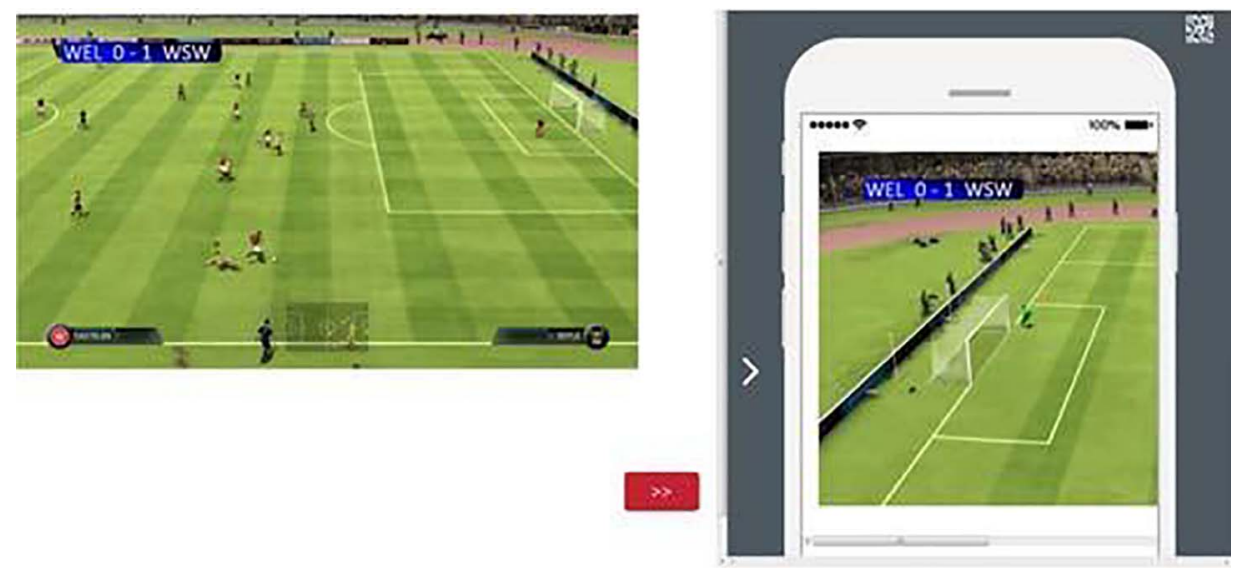

Figure 1. Screenshot example of soccer game for desktop (left) and mobile version (right) of the survey 
Table 1. Baseline and four inducements used in the experiment

\begin{tabular}{ll}
\hline Classes of incentive & \multicolumn{1}{c}{ Representative inducement* } \\
\hline No-inducement/baseline & Bet $\$ 4$ on your winning team! \\
Bonus bet & Receive a free bonus bet $(\$ 4)$ if your team is ahead at half-time! \\
Better odds/winnings & Boost your odds. Payout for winning bets is double! \\
Reduced risk & If your team wins the toss, but loses the game, get double your money back! \\
Cash rebate & $\$ 2$ cash back. Win or lose! \\
\hline
\end{tabular}

Note. *The text describing each inducement is the exact wording shown to participants.

Table 2. Risk/odds for each bet, expressed as required margin, for each game ${ }^{\mathrm{a}}$

\begin{tabular}{lcclll}
\hline Risk/odds & $\begin{array}{c}\text { Payoff per } \\
\text { dollar bet }\end{array}$ & $\begin{array}{c}\text { Payoff based on } \$ 4 \\
\text { wager (before incentives) }\end{array}$ & AFL & Cricket & Soccer \\
\hline Short & $\$ 1.90$ & $\$ 7.60$ & Outright win & Outright win & Outright win \\
Medium & $\$ 4.00$ & $\$ 16.00$ & +25 points & 5 wickets or 21 run margin & +2 goals \\
Long & $\$ 6.50$ & $\$ 26.00$ & +43 points & 8 wickets or 41 run margin & +3 goals \\
\hline
\end{tabular}

Note. AFL: Australian Football League.

${ }^{a}$ The winning margins illustrated in this table were based on a study of actual odds offered by bookmakers for roughly evenly matched teams. These margins were constructed to be within the range of what gamblers might expect from bookmakers, rather than precise figures. Our key consideration for the validity of the experiment was to create a situation where at least some players might be tempted to choose a more risky bet within each type of sport, while simultaneously remaining within the bounds of outcomes that would appear plausible to the participants.

based on our inspection of their typical payouts. However, the four inducement conditions had added \$ compensation based on betting outcomes, consistent with the incentive offered. Each incentivized outcome was constructed to have the same expected value (an extra \$2), either in cash or bets, to create an equivalency for enhancing the validity of later comparisons.

The Bonus bet is worth $\$ 2$ on average for participants because the teams are evenly matched for a win; therefore, the chance of the outcome (being ahead at halftime) is 50/50 (assuming no draw), so in the long run, the inducement is worth an average of $\$ 2$ per person. Better odds/winnings are worth $\$ 2$ on average for participants also because the teams are evenly matched for a win; therefore, half of the participants will have (in essence) an additional $\$ 4$ wagered on their bet, and the remaining half will have an additional $\$ 0$ wagered on the bet (see the section below on why this inducement was removed from Game 6). Reduced risk is worth $\$ 2$, on average, for participants because it is based on two contingencies happening, each with $50 \%$ chance of coming true. Thus, the overall chance of the inducement requirements being met is $25 \%$ and $25 \%$ of a doubling of the bet-size (\$8) is $\$ 2$. Finally, the Cash rebate is $\$ 2$ back for all participants, regardless of bet outcome, and is therefore, by definition, worth $\$ 2$ to everyone.

Game 6. One final game allowed participants to select among all five different types of incentives, including noincentive. This allowed us to understand the most attractive inducement for each participant, and potentially which specific inducements are particularly attractive to vulnerable groups.

During the soft launch, over half the sample selected the Better odds/winnings condition for their sixth game. While all inducements were set so that they had equal expectedvalue, players were not operating a betting account, per se, and thus, any winnings or inducements were not able to be used on subsequent bets. The experiment was designed in this way so that each bet was independent of previous bets. However, this meant that the Better odds/winnings inducement was more attractive because, even though its value was the same as for the other inducements (\$2), the highest total amount could potentially be won within the experiment with this incentive. This situation occurred because the value of the Better odds/winnings inducement was essentially "bet" as part of the original bet, and thus had the chance to be multiplied by the relevant odds (if successful), while the similar real-world inducements would need to be used in a subsequent bet. The Better odds/winnings inducement was, thus, removed as an option in the sixth game in the full launch. Accordingly, any results pertaining to Game 6 are only presented with the four inducements (No-inducement, Bonus bet, Reduced risk, and Cash rebate).

Finally, we asked players to rank order the inducements in the experiment from "most favorite" to "least favorite." This ranking allowed us to compare, for the entire sample, the concordance between the attractiveness of each inducement, and the risk they assumed when placing associated bets.

\section{Statistical analyses}

The dependent variable for Q1 was odds selected (short, medium, and long). The relationship between odds selected and inducement type was tested using a non-parametric Wilcoxon signed-rank test, and potential interactions with PGSI were tested with a cumulative link ordinal mixed analysis of variance (ANOVA) with planned contrasts. The inducement chosen in Game 6 was the dependent variable for Q2, tested using one-sample $\chi^{2}$ test, and the relationship with problem gambling severity was tested using multinomial logistic regression. The dependent variable for Q3 was attractiveness of the inducements, and differences between 
the inducements were tested with a Wilcoxon signed-rank test. An $\alpha$ of .05 was used throughout.

\section{Ethics}

The research described herein complies with the (Australian) National Statement on Ethical Conduct in Human Research (NHMRC). All participants gave informed consent and were informed they could withdraw at any time. Data were anonymized before analysis. The study was approved by Human Research Ethics Committee of Central Queensland University, clearance number Project \#H16/09-257.

\section{RESULTS}

The results are organized according to the three research questions.

\section{Q1: Do inducements influence the selection of longer odds bets?}

Wilcoxon signed-ranks test indicated that bettors chose longer odds with the Cash rebate inducement $(M=1.66$, $S D=0.61)$ compared to the No-inducement condition, $M=1.55, \quad S D=0.60, \quad Z=-2.664, \quad p<.05, \quad r=-.10$. Although all incentivized bets had higher mean risk, the other three individual inducements (Better odds/winnings, Bonus bet, and Reduced risk) did not significantly differ from the No-inducement condition on an individual basis. These findings were replicated using a cumulative link ordinal mixed ANOVA, incorporating main effects for condition and PGSI status. Other demographics, including age, gender, and personal/household income, showed no main effects, and were excluded from the model. We specified a second model incorporating an interaction between condition and PGSI category, which did not significantly improve the likelihood: log-likelihood ratio $(12)=12.27, p=.42$, ns. Accordingly, we concluded that there was no evidence for the proposition that PGs responded differently to the different inducement types.
A planned-contrast showed that the entire set of inducements, taken as a whole, showed significantly higher risktaking, by selection of longer odds, than the No-inducement condition, $p<.05$. Moreover, Figure 2 illustrates that the mean risk-choice is higher than the No-inducement condition for every incentivized condition.

\section{Q2: When given a choice, which inducements do sports} bettors select?

Excluding the Better odds/winning inducement (described above), the Bonus bet inducement was the most selected inducement for the sixth game, followed by the Noinducement condition, and then Reduced risk, and finally by Cash rebate. Many participants selected the No-inducement condition $(26.2 \%)$ for the sixth game, despite the fact that all of the incentivized conditions are objectively better by virtue of offering a $\$ 2$ in expected extra value.

However, a one-sample $\chi^{2}$ test found significant differences between the inducement type selected by participants for their sixth game based on their assigned game-type (i.e., AFL, soccer, or cricket), $\chi^{2}(3)=9.78, p<.05$. AFL and soccer bettors selected the Bonus bet inducement as their most preferred sixth game, whereas cricket bettors most frequently chose the Reduced risk inducement (Table 3).

We conducted a multinomial logistic regression to determine whether the selection made on the final game was dependent on problem gambling severity. The overall model fit was not a significant improvement over the null, LR = $2.11, p=.71$. Accordingly, we found no evidence that PGSI status influences choice of inducement.

\section{Q3: What inducements do participants rank as most attractive?}

Figure 3 illustrates the mean attractiveness ratings for all inducements on a 5-point Likert scale.

Overall, the most attractive condition was the Better odds/winning inducement and the least attractive was the No-inducement condition. There were no significant

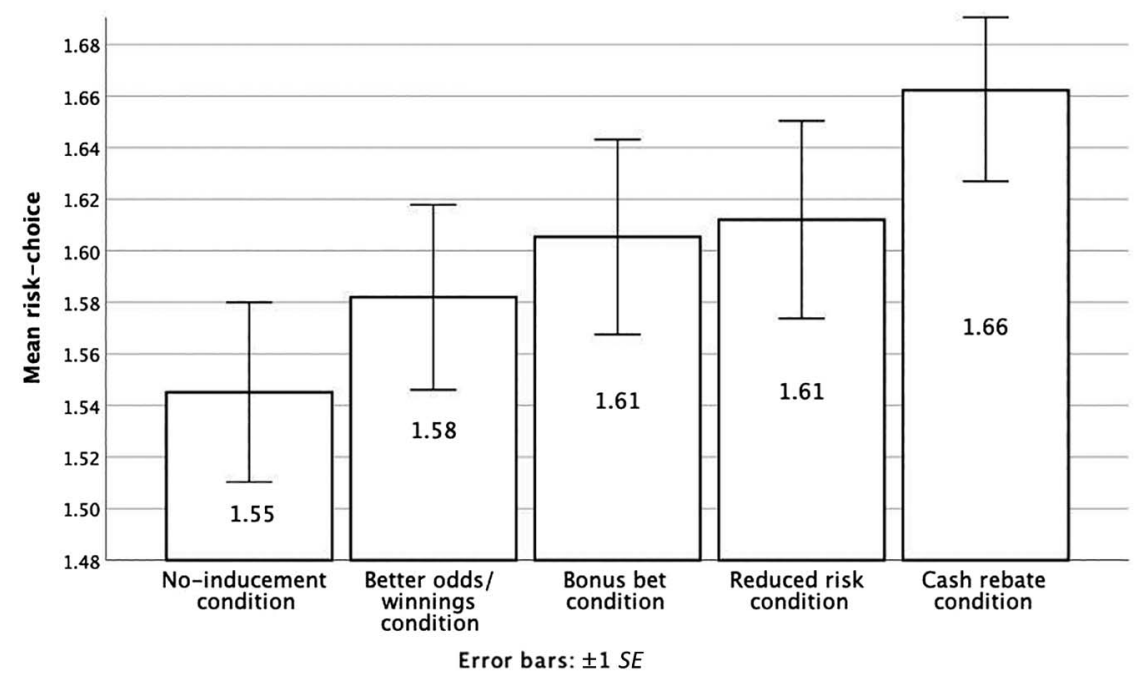

Figure 2. Mean ( $\pm 1 S E$ ) risk-choice for each condition (1-3 for short, medium, and long odds, respectively) 
Table 3. Frequency of inducement condition selected by participants for their sixth game

\begin{tabular}{lcccc}
\hline Condition & AFL $(n=89)$ & Soccer $(n=92)$ & Cricket $(n=90)$ & Total $(n=271)$ \\
\hline No-inducement/baseline & $27(30.3 \%)$ & $22(23.9 \%)$ & $22(24.4 \%)$ & $71(26.2 \%)$ \\
Bonus bet & $30(33.7 \%)$ & $31(33.7 \%)$ & $24(26.7 \%)$ & $85(31.4 \%)$ \\
Reduced risk & $19(21.3 \%)$ & $18(19.6 \%)$ & $29(32.2 \%)$ & $66(24.4 \%)$ \\
Cash rebate & $13(14.6 \%)$ & $21(22.8 \%)$ & $15(16.7 \%)$ & $49(18.1 \%)$ \\
\hline
\end{tabular}

Note. Better odds/winnings not included as an option in full launch and thus not analyzed. AFL: Australian Football League.

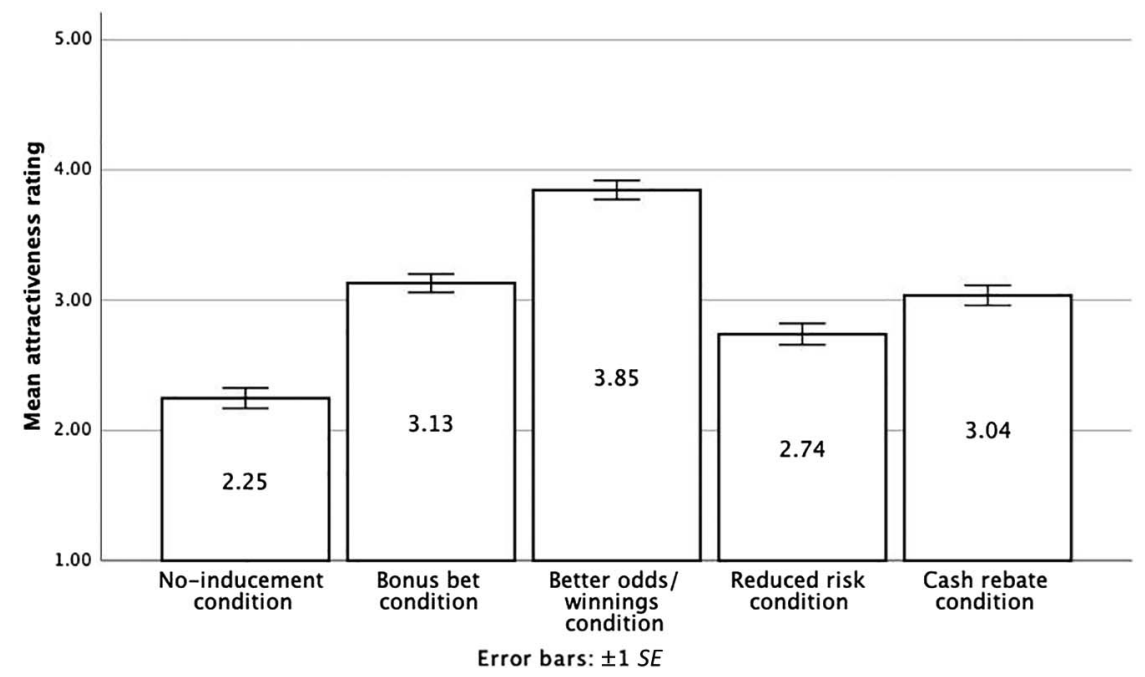

Figure 3. Mean $( \pm 1 S E)$ attractiveness ratings (1-5 Likert scale)

differences between PGSI groups in terms of rated attractiveness of any of these inducements, $p>.05$, ns.

Attractiveness of the inducements differed significantly, $\chi^{2}(4)=163.69, p<.001$. To further examine these differences, each of the inducements was compared against the No-inducement condition using Wilcoxon signed-rank tests. The No-inducement condition $(M=2.25, S D=1.34)$ was ranked significantly less attractive than the Bonus bet $(M=3.13, S D=1.22)$, Better odds/winnings $(M=3.85$, $S D=1.26)$, Reduced risk $(M=2.74, S D=1.41)$, and Cash rebate conditions $(M=3.04, S D=1.32)$.

\section{DISCUSSION AND CONCLUSIONS}

The study was an online experiment that aimed to find if the presence of betting incentives causes people to select riskier bets, and to find which incentives were preferred. Since incentives offer extra value to consumers, such value should lead to higher consumption, based on well-established tenets of microeconomic theory. However, we hypothesized that betting incentives may also induce gamblers to take extra risks with their bets in the form of selecting longer odds. Framing effects can have a significant influence on behavior. Often incentives appear to lessen risk rather than simply adding value to a transaction (Hing, Sproston, et al., 2017; Lopez-Gonzalez et al., 2018a; Lopez-Gonzalez \& Griffiths, 2017), and thus might encourage longer odds selection. In addition, incentives can appear to enhance the reward of winning and thereby justify longer odds betting if the winning outcome is more salient in comparison to the decreased odds of winning. Moreover, Newall's (2017) experimental research demonstrated that sports bettors typically overestimate the odds of winning when presented with complex bets. Incentives necessarily introduce some complexity into betting, which can impact on how gamblers perceive the value in the transaction. For these reasons, it is at least plausible to suggest that gamblers might see betting incentives as an opportunity to risk more.

\section{Bettors see incentives as attractive}

Participants perceived all bets with inducements (Bonus bets, Better odds/winnings, Reduced risk, and Cash rebate) as more attractive than bets without inducements. Certainly, this is a rational perception, since such bets offer extra value to the consumer that is (presumably) funded by the operator. In the pretest, the Better odds/winnings inducement was the overwhelming favorite selection for a sixth game incentivized bet. In the full sample, however, the Better odds/ winnings inducement was withdrawn to gain a better understanding of the attractiveness of the remaining inducements. AFL and soccer players chose the Bonus bet, whereas Cricket bettors selected the Reduced risk incentive the most often. These results reveal that the appeal of inducements is likely to be somewhat contextual, and may reflect the peculiarities of bettors on different sports and/or the structural characteristics of the sports. Overall, the experiment provides strong evidence of the attractiveness of wagering inducements, confirming results from previous 
self-report (Hing et al., 2014; Lopez-Gonzalez et al., 2018a; Lopez-Gonzalez \& Griffiths, 2017; Sproston, Hanley, Brook, Hing, \& Gainsbury, 2015) and conjoint studies (Hing, Vitartas, \& Lamont, 2017).

\section{Bettors choose long odds when offered an incentive}

Most importantly, and in line with predictions, people tended to choose longer odds bets when an inducement was present. All the inducement trials attracted higher average risk-taking than the No-inducement trial, although only the Cash rebate incentive individually showed a significantly greater risk profile.

\section{The behavioral effects of inducements are not unique to people with gambling problems}

No differences in results were observed between PGSI risk groups. Thus, the results indicate that wagering inducements increase the appeal of betting and the tendency to place riskier bets among all gambler groups (NPG, LR, MR, and PG) - not just vulnerable gamblers. This finding contrasts with previous conclusions that PGs are typically most influenced by gambling marketing in general (Binde, 2014) and by wagering marketing in particular (Hing, Russell, et al., 2017; Sproston et al., 2015), and may indicate a tendency of PGs to self-report greater influence. The lack of differences between PGSI risk groups may also reflect the range of incentives explored, including incentives with the universally appealing feature of appearing to reduce downside risk. Finally, wagering incentives can appeal to players through the many cognitive bias and heuristics that are common among regular gamblers, and not just gamblers with problems (Delfabbro, 2004; Myrseth, Brunborg, \& Eidem, 2010), and can lead to excessive spending and harm across all PGSI risk categories (Browne \& Rockloff, 2018, Browne et al., 2017).

\section{Consequences of wagering incentives on bettors and the industry}

Wagering operators tend to incentivize complex bets whose odds are difficult to estimate (such as those with combined contingencies), and risky bets, which are associated with low odds of winning and increased gambling losses and operator profits (Newall, 2015). The tendency to choose bets with longer odds when accompanied by an inducement, as found in this study, necessarily increases the proportion of bettors likely to lose the bet, with only a smaller proportion of gamblers enjoying higher wins - compared to bets with shorter odds. That is, betting on riskier propositions increases the number of losing bettors. The fact that inducements encourage more risk-taking is non-obvious, and practically very important. In a commercial environment where the expected returns from gambling are negative for the gambler, high-risk bets lead to greater gambling losses. It may be that inducements effectively pay for themselves where they encourage riskier bets, and thereby amplify operators' profits and gamblers' losses. Moreover, in some jurisdictions, the wins on incentivized bets have playthrough conditions that require any winning amounts be transformed into future bets (Hing, Sproston, et al., 2017), and these play-through conditions appear to be underestimated by consumers (Hing, Browne, et al., 2018). Complex play-through conditions can subtract value from incentives and even require stakes of new money that negate their value.

\section{Limitations}

The experimental nature of the study has high internal validity, but necessarily at the sacrifice of some elements of external validity. Gambling on simulated matches may not have been perceived by participants to operate similarly to gambling on "real" matches. Real matches operate according to team skill and other factors, whereas the simulated games in the experiment had preconfigured outcomes produced by the researchers. Although we attempted to produce highfidelity highlight reels for our games, participants were aware that these games were not "real" and thus their judgment about appropriate risk taking or likely outcomes may have reflected considerations that would not operate in a naturalistic setting involving real matches.

The size of bets on individual matches, at $\$ 4$, was typically below the average bet size for real matches and commercial betting (Gainsbury \& Russell, 2015). Although many gamblers bet small amounts, some bettors, and particularly high-intensity gamblers, might typically wager much larger amounts. Nevertheless, the tendency of participants to seek lower-risk bets on average, rather than the highest possible long odds bets, provides some confidence that they treated these bets with some seriousness with respect to protecting their bankroll.

The inducements, at an expected value of $\$ 2$, were large relative to bet size. More often, real inducements have a lower expected value relative to the size of the bet. The strength of each inducement was purposefully set to allow us find significant effects for inducements if they exist. Real incentives might have a weaker effect on players if their action is proportional to the size of the bet.

Finally, gamblers often shop multiple bookmakers for the best incentives, whereas we only offered one incentive type for each of the first five games. Risk choice may be altered when several incentives are considered simultaneously, and only one perceived "best" incentive is chosen.

\section{CONCLUSIONS}

This experiment found that incentivized bets on wagering are likely to influence gamblers to choose long odds/highrisk bets. There is no definitive logical reason that gamblers should select longer odds when a bet is incentivized. Although the extra value represented by incentives should logically increase consumption, it should not necessarily increase attitudes toward risk. The medium to long-run consequence of risky betting is to produce a greater number of gamblers in a losing position, which can contribute to player harm. Finally, assuming that the incentivized products are not, actuarially, priced well in comparison to nonincentivized bets, it would also enable a greater net transfer of wealth from gamblers to gambling operators. 
Funding sources: This study was funded by the Victorian Responsible Gambling Foundation: Tender for the project entitled "Effects of wagering marketing on vulnerable adults," \#1-16.

Authors' contribution: All authors designed the study and conducted the survey and data collection. MJR and MB led the overall project. AMTR programmed the survey with the assistance of a research assistant. MB conducted the data analysis. All authors were involved in the drafting of the manuscript, read, and commented on the manuscript and approved it for submission.

Conflict of interest: MJR has received research grants from the Queensland Treasury, the Victorian Treasury, the Victorian Responsible Gambling Foundation, the New Zealand Ministry of Health, the NSW Dept of Industry and Trade, the Department of Social Services, the Alberta Gambling Research Institute, and Gambling Research Australia. MB has received grants from the Victorian Responsible Gambling Foundation, the New Zealand Ministry of Health the NSW Dept of Industry and Trade, the Department of Social Services, the Alberta Gambling Research Institute, and Gambling Research Australia. AMTR has received research funding from the Victorian Responsible Gambling Foundation, Queensland Justice and Attorney-General, Gambling Research Australia, National Association for Gambling Studies, Australian Communications and Media Authority, and the Alberta Gambling Research Institute. He has received industry funding for an evaluation of problem gambling among casino employees from Echo/Star Entertainment Group. He is also affiliated with the University of Sydney. NR has received research funds from the Victorian Responsible Gambling Foundation, Gambling Research Australia, Australian Department of Social Services, Alberta Gambling Research Institute, the Australian Gambling Research Centre, the Queensland, New South Wales, Victorian and South Australian Governments, the Australian Research Council, and Australia's National Research Organisation for Women's Safety. She has also received consultancy funds from Echo Entertainment and Sportsbet and an honorarium from Singapore Pools for membership of its International Advisory Committee. All authors declare no conflict of interest in relation to this manuscript.

\section{REFERENCES}

Binde, P. (2014). Gambling advertising: A critical research review. London, UK: Responsible Gambling Trust. Retrieved from https://about.gambleaware.org/media/1165/binde_rgt_report_ gambling_advertising_2014_final_color_115p.pdf

Browne, M., Hing, N., Russell, A. M. T., Thomas, A., \& Jenkinson, R. (2019). The impact of exposure to wagering advertisements and inducements on intended and actual betting expenditure: An ecological momentary assessment study. Journal of Behavioral Addictions, 8(1), 146-156. doi:10.1556/2006. 8.2019 .10
Browne, M., Rawat, V., Greer, N., Langham, E., Rockloff, M. R., \& Hanley, C. (2017). What is the harm? Applying a public health methodology to measure the impact of gambling problems and harm on quality of life. Journal of Gambling Issues, 36, 28-50. doi:10.4309/jgi.2017.36.2

Browne, M., \& Rockloff, M. J. (2018). Prevalence of gamblingrelated harm provides evidence for the prevention paradox. Journal of Behavioral Addictions, 7(2), 410-422. doi:10.1556/ 2006.7.2018.41

Delfabbro, P. (2004). The stubborn logic of regular gamblers: Obstacles and dilemmas in cognitive gambling research. Journal of Gambling Studies, 20(1), 1-21. doi:10.1023/B: JOGS.0000016701.17146.d0

Ferris, J. A., \& Wynne, H. J. (2001). The Canadian Problem Gambling Index: Final report. Ottawa, Canada: Canadian Centre on Substance Abuse.

Friedman, M. (1949). The marshallian demand curve. The Journal of Political Economy, 57(6), 463-495. doi:10.1086/256879

Gainsbury, S. M., \& Russell, A. (2015). Betting patterns for sports and races: A longitudinal analysis of online wagering in Australia. Journal of Gambling Studies, 31(1), 17-32. doi:10.1007/s10899-013-9415-4

Hing, N., Browne, M., Russell, A. M. T., Greer, N., Thomas, A., Jenkinson, R., \& Rockloff, M. (2018). Where's the bonus in bonus bets? Assessing sports Bettors' comprehension of their true cost. Journal of Gambling Studies. Advance online publication. doi:10.1007/s10899-018-9800-0

Hing, N., Cherney, L., Gainsbury, S. M., Lubman, D. I., Wood, R. T., \& Blaszczynski, A. (2014). Maintaining and losing control during Internet gambling: A qualitative study of gamblers' experiences. New Media \& Society, 17(7), 1075-1095. doi:10.1177/1461444814521140

Hing, N., Russell, A. M. T., Lamont, M., \& Vitartas, P. (2017). Bet anywhere, anytime: An analysis of Internet sports bettors' responses to gambling promotions during sports broadcasts by problem gambling severity. Journal of Gambling Studies. Advance online publication. doi:10.1007/s10899-017-9671-9

Hing, N., Russell, A. M. T., Li, E., \& Vitartas, P. (2018). Does the uptake of wagering inducements predict impulse betting on sport? Journal of Behavioral Addictions, 7(1), 146-157. doi:10.1556/2006.7.2018.17

Hing, N., Russell, A. M. T., Thomas, A., \& Jenkinson, R. (2019). Wagering advertisements and inducements: Exposure and perceived influence on betting behaviour. Journal of Gambling Studies. Advance online publication. doi:10.1007/s10899-01809823-y

Hing, N., Sproston, K., Brading, R., \& Brook, K. (2015). Review and analysis of sports and race betting inducements. Melbourne, Australia: Victorian Responsible Gambling Foundation.

Hing, N., Sproston, K., Brook, K., \& Brading, R. (2017). The structural features of sports and race betting inducements: Issues for harm minimisation and consumer protection. Journal of Gambling Studies, 33(2), 685-704. doi:10.1007/s10899016-9642-6

Hing, N., Vitartas, P., \& Lamont, M. (2017). Understanding persuasive attributes of sports betting advertisements: A conjoint analysis of selected elements. Journal of Behavioral Addictions, 6(4), 658-668. doi:10.1556/2006.6.2017.062

Lopez-Gonzalez, H., Estévez, A., \& Griffiths, M. D. (2017). Marketing and advertising online sports betting: A problem 
gambling perspective. Journal of Sport \& Social Issues, 41(3), 256-272. doi:10.1177/0193723517705545

Lopez-Gonzalez, H., Estévez, A., \& Griffiths, M. D. (2018a). Controlling the illusion of control: A grounded theory of sports betting advertising in the UK. International Gambling Studies, 18(1), 39-55. doi:10.1080/14459795.2017.1377747

Lopez-Gonzalez, H., Estévez, A., \& Griffiths, M. D. (2018b). Internet-based structural characteristics of sports betting and problem gambling severity: Is there a relationship? International Journal of Mental Health and Addiction. Advance online publication. doi:10.1007/s11469-018-9876-x

Lopez-Gonzalez, H., \& Griffiths, M. D. (2017). "Cashing out" in sports betting: Implications for problem gambling and regulation. Gaming Law Review, 21(4), 323-326. doi:10.1089/ $\operatorname{glr} 2.2017 .2144$

Myrseth, H., Brunborg, G. S., \& Eidem, M. (2010). Differences in cognitive distortions between pathological and non- pathological gamblers with preferences for chance or skill games. Journal of Gambling Studies, 26(4), 561-569. doi:10.1007/s10899-010-9180-6

Newall, P. W. S. (2015). How bookies make your money. Judgement and Decision Making, 10(3), 225-231.

Newall, P. W. S. (2017). Behavioral complexity of British gambling advertising. Addiction Research \& Theory, 25(6), 505-511. doi:10.1080/16066359.2017.1287901

Russell, A. M. T., Hing, N., Browne, M., \& Rawat, V. (2018). Are direct messages (texts and emails) from wagering operators associated with betting intention and behaviour? An ecological momentary assessment study. Journal of Behavioral Addictions, 7(4), 1079-1090. doi:10.1556/2006. 7.2018.99

Sproston, K., Hanley, C., Brook, K., Hing, N., \& Gainsbury, S. M. (2015). Marketing of sports betting and racing. Melbourne, Australia: Gambling Research Australia. 